\title{
Malignant peripheral nerve sheath tumors of the trigeminal nerve: a systematic review of 36 cases
}

\author{
Richard F. Schmidt, B.A., ${ }^{1}$ Frederick Yick, B.A., ${ }^{1}$ Zain Boghani, B.S., ${ }^{1}$ \\ JeAn Anderson Eloy, M.D., ${ }^{1-3}$ AND JAMES K. LiU, M.D. ${ }^{1-3}$
}

Departments of ${ }^{1}$ Neurological Surgery and ${ }^{2}$ Otolaryngology-Head and Neck Surgery, ${ }^{3}$ Center for Skull Base and Pituitary Surgery, Neurological Institute of New Jersey, University of Medicine and Dentistry of New Jersey-New Jersey Medical School, Newark, New Jersey

\begin{abstract}
Object. Malignant peripheral nerve sheath tumors (MPNSTs) are a rare form of malignancy arising from the Schwann cells of peripheral nerves. MPNSTs of the trigeminal nerve are exceptionally rare, with only a handful of reports in the literature. These tumors are typically very aggressive, resulting in significant patient morbidity and a generally grim prognosis. Most current reports suggest that radical resection with radiation therapy offers the best benefit. In this study, the authors systematically reviewed the world English-language literature on MPNSTs of the trigeminal nerve to analyze the presentations, treatment options, and outcomes for patients with this disease.

Methods. A literature search for MPNSTs of the trigeminal nerve confined to nonanimal, English-language articles was conducted utilizing the PubMed database, with additional cases chosen from the references of selected articles. Only cases of confirmed MPNSTs of the trigeminal nerve or its peripheral branches, based upon surgical, pathological, or radiological analysis, were included.

Results. From the literature search, 29 articles discussing 35 cases of MPNSTs of the trigeminal nerve were chosen. With the addition of 1 case from their own institution, the authors analyzed 36 cases of trigeminal MPNSTs. The average age of onset was 44.6 years. These tumors were more commonly seen in male patients $(77.1 \%)$. The gasserian ganglion was involved in $36.1 \%$ of the cases. Of the cases in which the nerve distribution was specified (n $=25)$, the mandibular branch was most commonly involved $(72.0 \%)$, followed by the maxillary branch $(60.0 \%)$ and the ophthalmic branch (32.0\%), with $44.0 \%$ of patients exhibiting involvement of 2 or more branches. Altered facial sensation and facial pain were the 2 most commonly reported symptoms, found in $63.9 \%$ and $52.8 \%$ of patients, respectively. Mastication difficulty and diplopia were seen in $22.2 \%$ of patients, facial weakness was seen in $19.4 \%$, and hearing loss was present in $16.7 \%$. With regard to the primary treatment strategy, $80.6 \%$ underwent resection, $16.7 \%$ underwent radiation therapy, and $2.9 \%$ received chemotherapy alone. Patients treated with complete resection followed by postoperative radiation therapy had the most favorable outcomes, with no patients showing evidence of disease recurrence with a mean follow-up of 34.6 months. Patients treated with incomplete resection followed by postoperative radiation therapy had more favorable outcomes than patients treated with incomplete resection without radiation therapy or radiation therapy alone.

Conclusions. Trigeminal MPNSTs most commonly present as altered facial sensation or facial pain, although they exhibit a number of other clinical manifestations, including the involvement of other cranial nerves. While a variety of treatment options exist, due to their highly infiltrative nature, aggressive resection followed by radiation therapy appears to offer the greatest chance of recurrence-free survival.
\end{abstract} (http://thejns.org/doi/abs/10.3171/2012.11.FOCUS12292)

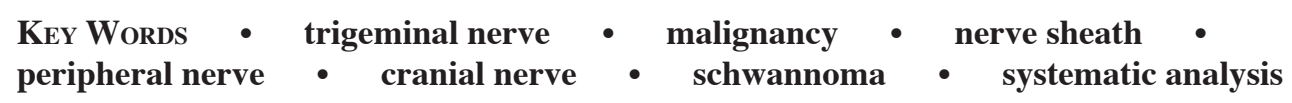

$\mathrm{M}$ ALIGNANT peripheral nerve sheath tumors (also known as neurofibrosarcomas or malignant schwannomas) are extremely rare sarcomas arising from the Schwann cells of peripheral nerves. ${ }^{5,12}$ These tumors have an incidence of approximately $0.001 \%$ in the general population ${ }^{13}$ and tend to present in older patients. ${ }^{12}$ Individuals with a family history of NF1 are

\footnotetext{
Abbreviations used in this paper: MPNST = malignant peripheral nerve sheath tumor; $\mathrm{NF}=$ neurofibromatosis; $\mathrm{NF1}=\mathrm{NF}$ Type 1 ; NF2 = NF Type 2.
}

reported to have an increased risk of developing MPNSTs in their lifetime. ${ }^{9}$ However, this association is not seen in MPNSTs of the cranial nerves. ${ }^{1,33}$ MPNSTs are typically found in the trunk and extremities and are not commonly seen in the head and neck region. ${ }^{31}$ MPNSTs of the cranial nerves are an exceptionally rare manifestation of this disease, as most schwannomas of the cranial nerves are benign. $1,3,21,33$ MPNSTs of the trigeminal nerve are exceedingly rare with very few existing reports in the literature. In this study, we performed a systematic review of 36 cases of MPNSTs of the trigeminal nerve and analyzed the 
clinical presentation, tumor distribution, treatment strategies, and outcomes for patients with this disease.

\section{Methods}

\section{Search Strategy}

A literature search for MPNSTs of the trigeminal nerve was conducted utilizing the PubMed database. The search was confined to nonanimal, English-language articles. The following search terms were used: "trigeminal AND sheath tumor," "trigeminal AND malignant peripheral nerve sheath tumor," "trigeminal AND malignant," "trigeminal AND nerve sheath tumor," "trigeminal AND neural sheath tumor," "trigeminal AND malignant tumor," "trigeminal AND malignant schwannoma," "trigeminal AND neurofibrosarcoma," and "trigeminal AND neurosarcoma." The references of included articles were searched for any additional pertinent studies.

\section{Inclusion Criteria}

We included all English-language articles-case reports, case illustrations, and case series. Relevant articles were determined by identifying reports of patients with confirmed MPNSTs of the trigeminal nerve. Patients were included in our study if specific trigeminal involvement was reported, either by specific declaration of trigeminal nerve involvement or description of anatomical distributions specific to trigeminal nerve distributions (for example, foramen ovale). Papers discussing tumors based on location with an unspecified nerve origin or nonspecific anatomical structures (for example, maxillary sinus MPNST) were not included in the study. One patient was reported as having bilateral MPNSTs that were diagnosed and treated separately during 2 separate time periods with different treatment regimens. As such, these lesions have been incorporated into our review as 2 separate cases, except in the analyses of sex and NF status. The selected reports were reviewed, and pertinent data, including age, sex, tumor location, clinical symptoms, method of treatment, recurrence, duration of follow-up (months), and disease status at final follow-up, were obtained when available.

\section{Exclusion Criteria}

Articles that were not written in English and animal studies were excluded during the PubMed search. Articles pertaining to anatomy, anesthesia, biochemistry, natural history, other disease, other tumors, pathology, pharmacology, radiology, and treatments/techniques not pertaining to trigeminal MPNSTs were excluded.

\section{Data Extraction}

All data were extracted by 3 independent authors, and any discrepancies were addressed following discussion. Tumor location was determined primarily on the basis of surgical or autopsy exploration. Radiological studies were used to determine tumor location only if findings specific to a certain tumor distribution were reported (for example, erosion of the foramen ovale by an enhancing lesion would indicate involvement of the mandibular divi- sion of the trigeminal nerve [V3]). Clinical presentation or distribution of symptoms (for example, pain or sensory disturbance in a specific distribution) was not used as a criterion for determining location, as we felt that this may not accurately depict exactly where the tumor occurred. "Not available" was also assigned to certain cases if involvement of the gasserian ganglion was specified but not the other nerve distributions. Tumors affecting peripheral and terminal branches of the trigeminal nerve were included when involvement of these nerves was specified. Clinical presentation was determined on the basis of the reported signs or symptoms.

\section{Data Analysis}

Microsoft Excel was used for data aggregation and analysis.

\section{Results}

Searching the PubMed database using the key words listed above identified 175 studies (Fig. 1). Exclusion criteria included other tumor (85 articles), treatments/techniques not pertaining to MPNSTs (27), radiology (10), other disease (9), natural history (7), pathology (4), biochemistry (3), pharmacology (3), anatomy (2), and anesthesia (1). After applying the aforementioned criteria, 24 articles were included in the systematic review. A search of the references of the 24 articles yielded 5 additional pertinent articles. These 29 articles provided 35 cases, to which we added 1 additional case from our institution. ${ }^{1,2,4-8,10,11,14-21,24,26-30,32-37}$ The complete results of our analysis of 36 cases are detailed in Table 1.

\section{Patient Demographics}

The patients' ages ranged from 8 to 71 years (mean 44.6 years). Eight patients were female (22.9\%) and 27 patients were male (77.1\%). Neurofibromatosis Type 1 was present in 1 patient $(2.9 \%)$, absent in 20 patients $(57.1 \%)$, and not assessed in 14 patients (40.0\%). Neurofibromatosis Type 2 was present in 1 patient $(2.9 \%)$, absent in 5 patients $(14.3 \%)$, and not assessed in 29 patients $(82.9 \%)$. Eleven cases $(30.6 \%)$ involved right-sided lesions, and 25 cases $(69.4 \%)$ involved left-sided lesions. Gasserian ganglion involvement was reported in 13 cases $(36.1 \%)$, including those in which the peripheral nerve involvement was not assessed. In total, the distribution of involved trigeminal nerve branches was not specified in 11 cases (30.6\%). Of the remaining 25 cases for which peripheral branch distributions were specified, $8(32.0 \%)$ had involvement of the ophthalmic branch (V1), 15 (60.0\%) had involvement of the maxillary branch (V2), and 18 (72.0\%) had involvement of the mandibular branch (V3). Of these cases, 11 $(44.0 \%)$ had involvement of more than one branch, with 6 $(24.0 \%)$ having involvement of 2 branches, and 5 (20.0\%) involvement of all 3 branches.

\section{Clinical Presentation}

Clinical presentation was reported in all cases. The most common presenting symptom was altered facial sensation, defined as any change in facial sensorium other than pain, and was present in 23 cases $(63.9 \%)$. Facial pain 


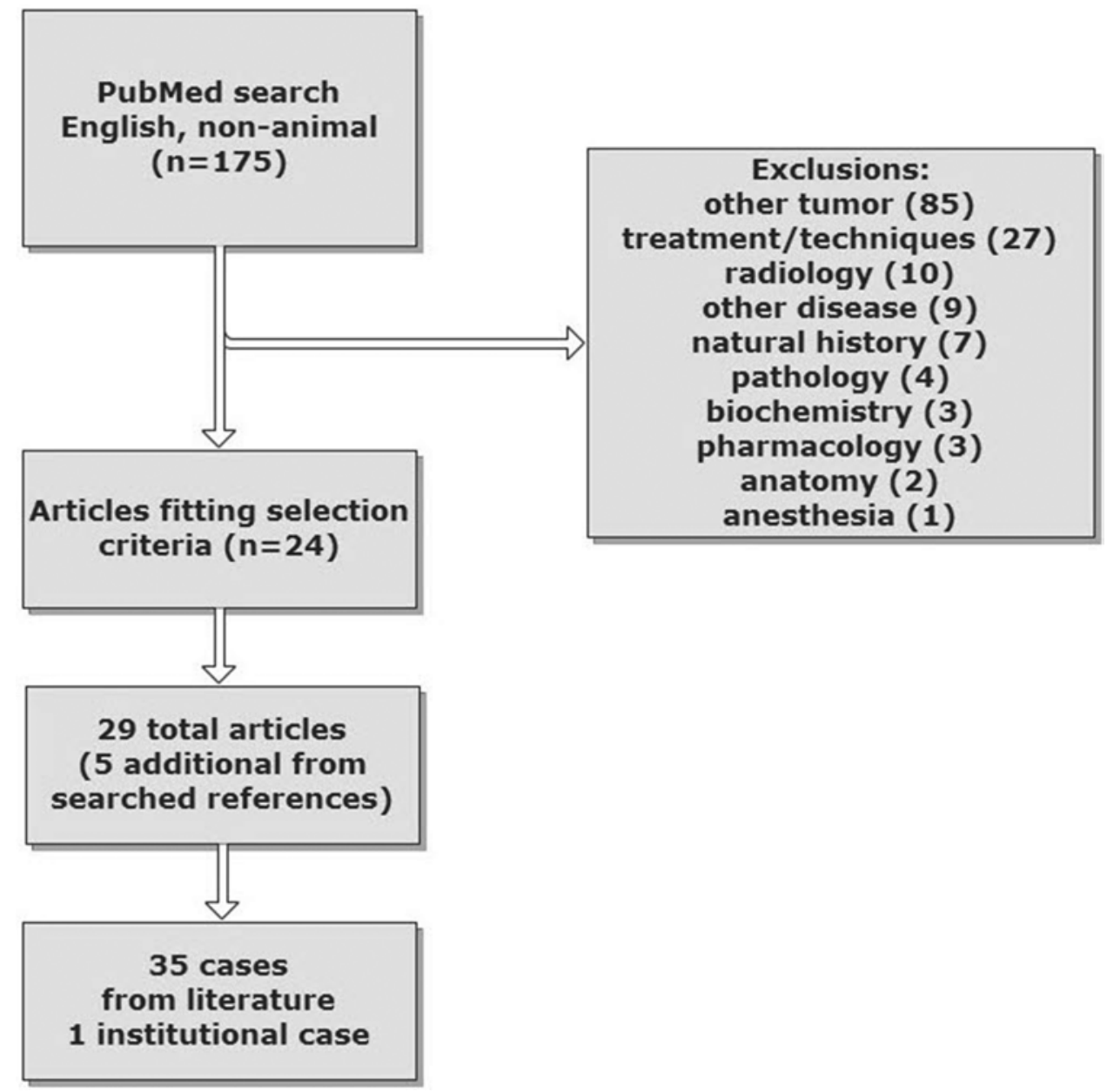

FIG. 1. Schematic for inclusion and exclusion of articles into the systematic analysis. The literature search yielded 29 studies of 35 cases of trigeminal MPNSTs. With the addition of the case from our institution, we obtained data from a total of 36 cases.

was the next most common symptom, present in 19 cases $(52.8 \%)$. Mastication difficulty, defined as any weakness or palsy of the muscles of mastication on presentation or physical examination, was present in 8 cases $(22.2 \%)$. Headache was seen in 5 cases $(13.9 \%)$. Involvement of other cranial nerves was also seen in a number of cases. Diplopia was present in 8 cases $(22.2 \%)$, and facial weakness was present in 7 (19.4\%). Hearing loss was present in 6 cases $(16.7 \%)$. Gustatory disturbance was present in 3 cases $(8.3 \%)$, and decreased visual acuity was present in $2(5.6 \%)$. Olfactory disturbance was present in 1 case $(2.9 \%)$. A number of other symptoms were also reported. Loose teeth were found in 2 cases $(5.6 \%)$ due to tumor involvement of the peripheral branches of V3. Other symptoms were reported only once (2.9\%), including epistaxis, nasal obstruction, facial fasciculations, ataxia, nausea and vomiting, decreased consciousness, ptosis, hemiparesis, tinnitus, phonophobia, and nocturnal breathing difficulty.

\section{Treatment Approaches}

The primary treatment intervention was reported in all 36 cases. Surgery was chosen as the primary intervention in 29 cases $(80.6 \%)$. In 9 cases $(25.0 \%)$ the lesions were treated with surgery alone, in 17 cases $(47.2 \%)$ they were treated with surgery and postoperative radiation treatment, and in 3 cases $(8.3 \%)$ surgery was performed but the authors did not specify whether radiation therapy was also administered or not. In 6 cases (16.7\%) radiation therapy was used as the sole primary treatment. The average dose of radiation when radiation therapy was used as a primary treatment was $5630 \mathrm{cGy}$. When specified (n $=14$ ), the average dose of radiation given as postoperative radiation therapy was $5940 \mathrm{cGy}$. Chemotherapy was given as part of the initial therapy in 4 cases (11.1\%), and it was given as the sole therapy in 1 case $(2.9 \%)$. It is important to note that the patient treated with chemotherapy alone was also the only patient with a reported history of NF2 and had massive systemic disease with MPNSTs of multiple nerves at the time of diagnosis. Of the 29 cases in which surgery was performed $(n=29)$, complete resection was accomplished in 7 (24.1\%), incomplete resections were reported in $19(65.5 \%)$, and the extent of resection was not specified in $3(10.3 \%)$.

\section{Outcomes}

Of the 36 cases in this study, 29 cases had complete details of the primary treatment as well as follow-up in- 
R. F. Schmidt et al.

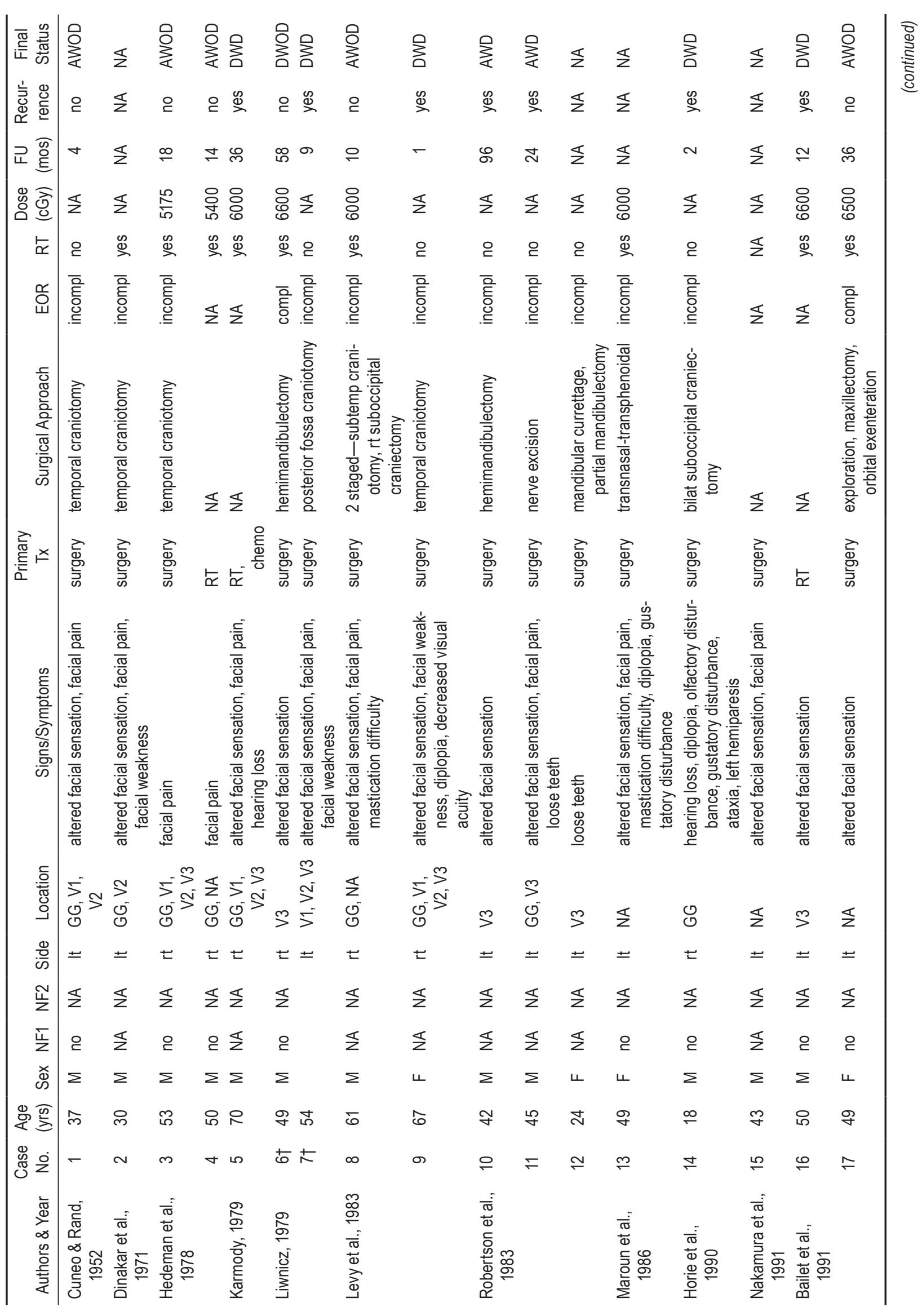

Neurosurg Focus / Volume 34 / March 2013 


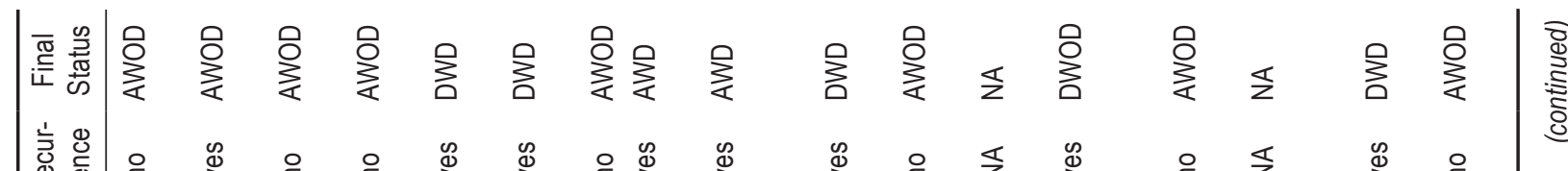

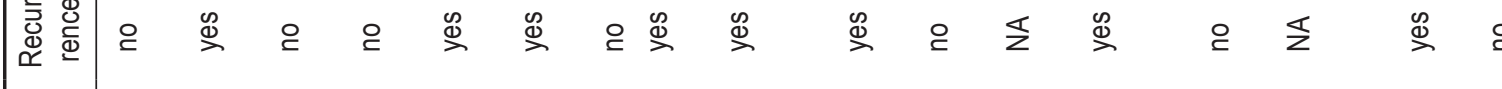
ฟ药

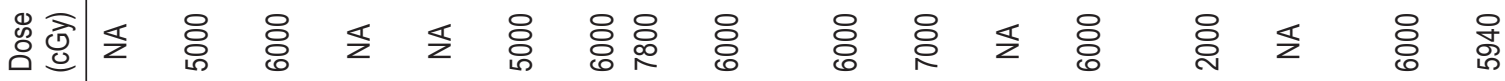

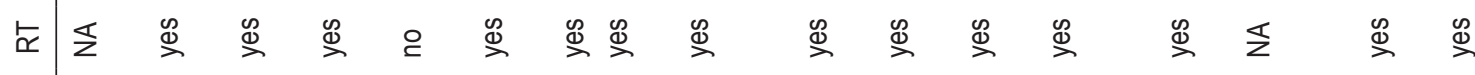

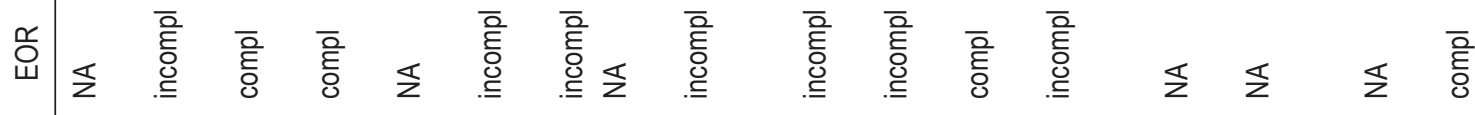

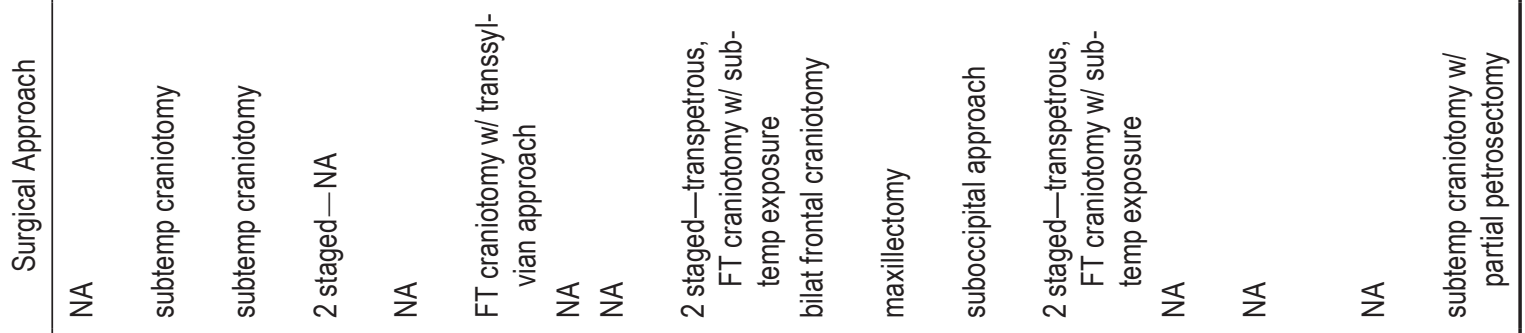

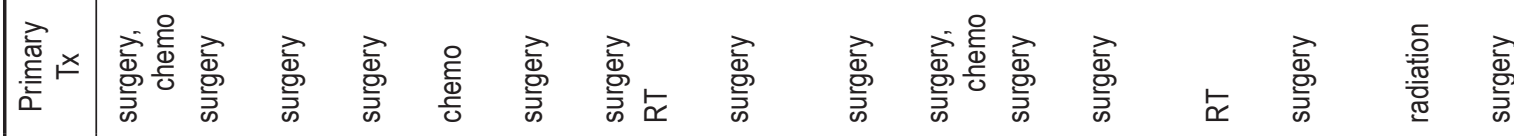

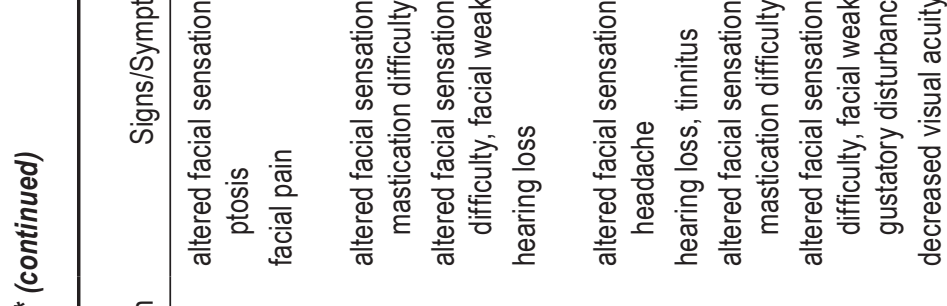

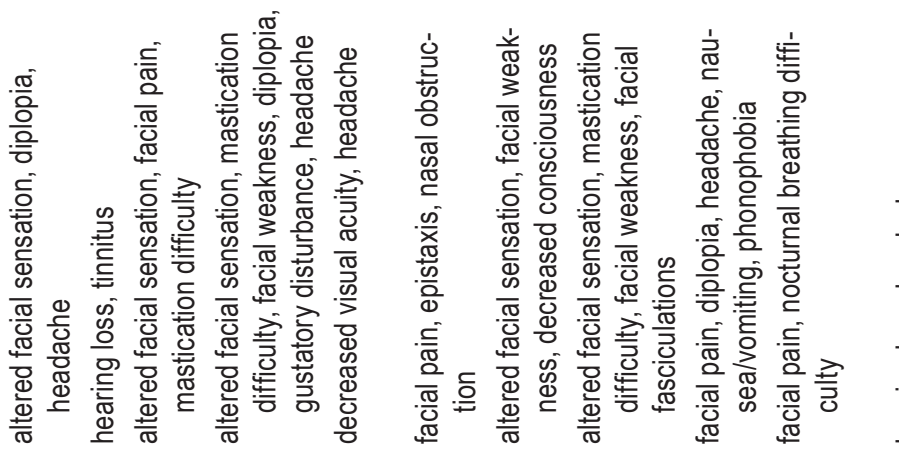

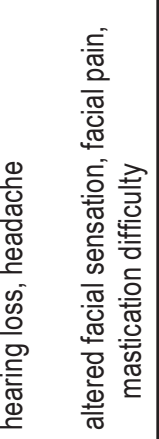

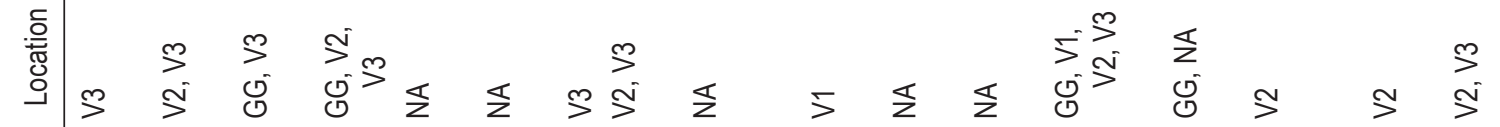
离 $= \pm \pm \pm \pm \pm \pm \pm \pm \pm \pm \pm \pm \pm= \pm$

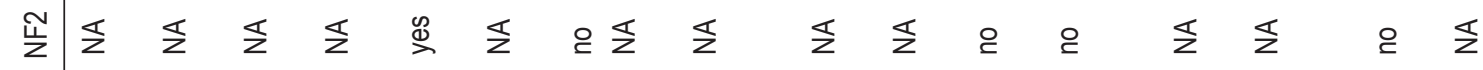

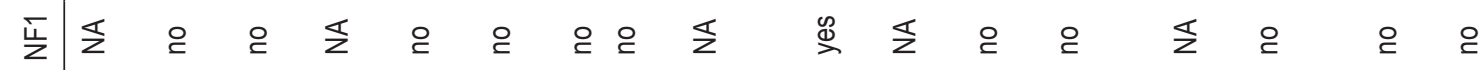

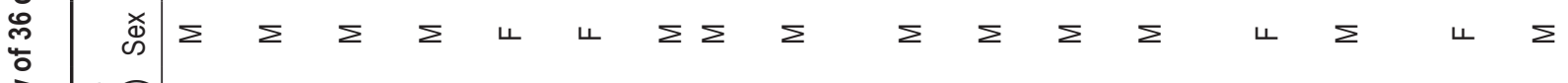

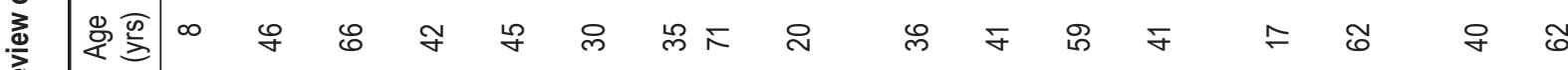

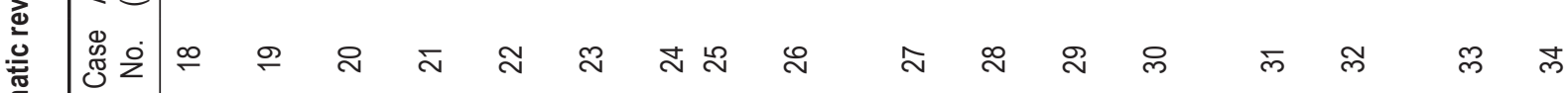

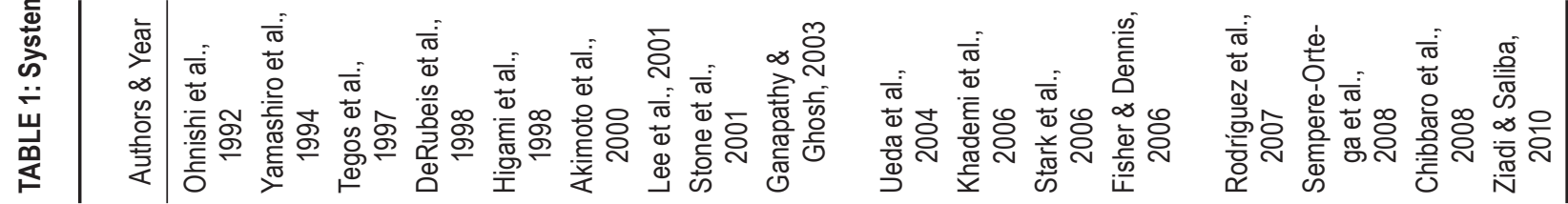




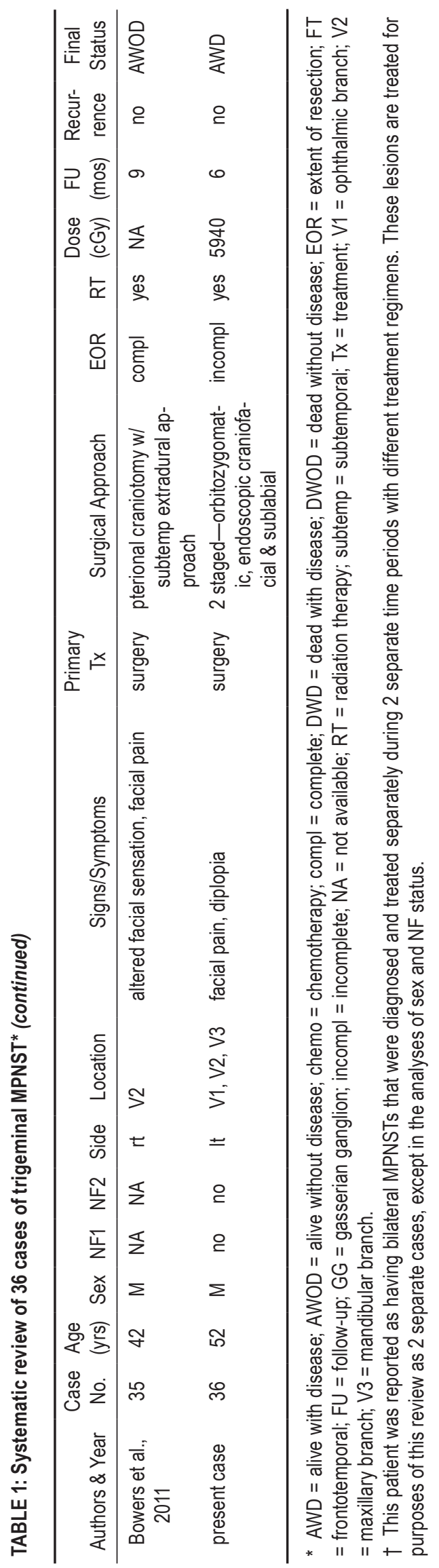

formation assessing patient outcome. Primary treatments were analyzed based on the extent of resection, the use of postoperative radiation therapy, or if the patient received radiation or chemotherapy as the primary treatment. In 6 $(20.7 \%)$ of these 29 cases, the primary treatment was complete resection with postoperative radiation, in 10 (34.5\%) it was incomplete resection with radiation therapy, in 6 (20.7\%) incomplete resection was performed without radiation therapy, in $6(20.7 \%)$ radiation therapy was administered as the sole treatment, and in 1 case $(3.4 \%)$ the treatment was chemotherapy alone.

To compare outcomes, overall recurrence was determined as well as the final status at the most recent follow-up. Recurrence was defined as the presence of any disease progression or metastasis throughout the entire postintervention period. Final outcomes were determined as the patient and disease status at the latest follow-up and were reported as "alive with disease" (AWD), "alive without disease" (AWOD), "dead with disease" (DWD), and "dead without disease" (DWOD). Patients were reported as being "with disease" if there was evidence of tumor recurrence or progression at last follow-up. Additionally, if the patient died in the perioperative or immediate postoperative period for a reoperation of recurrent disease, the status was DWD, even if total resection was accomplished. Follow-up times for these cases ranged from 1 to 96 months (average 24.4 months).

In the cases in which the primary treatment was complete resection with radiation therapy $(n=6)$, there was no evidence of recurrence. At the most recent followup (average 34.6 months, range 9-60 months), 5 patients $(83.3 \%)$ were AWOD and 1 (16.7\%) was DWOD. In the cases in which incomplete resection was performed and was followed by radiation treatment $(\mathrm{n}=10), 5(50.0 \%)$ had evidence of recurrence. At last follow-up (average 24.7 months, range 5-84 months) $2(20.0 \%)$ patients were AWD, $5(50.0 \%)$ were AWOD, 2 (20.0\%) were DWD, and $1(10.0 \%)$ was DWOD. In the cases in which incomplete resection was performed without radiation treatment $(\mathrm{n}=$ 6), 5 patients $(83.3 \%)$ showed evidence of recurrence. At last follow-up (average 22.7 months, range 1-96 months) $2(33.3 \%)$ were AWD, 1 (16.7\%) was AWOD, and 3 $(50.0 \%)$ were DWD. In the cases treated with radiation therapy alone $(n=6), 4$ patients $(66.7 \%)$ had evidence of recurrence. At last follow-up (average 21.3 months, range 9-36 months), 1 (16.7\%) patient was AWD, 2 (33.3\%) were AWOD, and $3(50.0 \%)$ were DWD. The 1 patient who was treated with chemotherapy had evidence of recurrence and was DWD at 4 months' follow-up.

\section{Illustrative Case}

This 52-year-old man presented to our department complaining of left-sided facial pain, left-sided facial swelling, and diplopia from partial left CN III, IV, and VI palsies. The facial pain was distributed along the V2 and V3 divisions of the trigeminal nerve. The patient was otherwise neurologically intact with no other complaints. Magnetic resonance imagining demonstrated an enhancing mass in the left cavernous sinus and Meckel cave with 


\section{Malignant peripheral nerve sheath tumors of the trigeminal nerve}
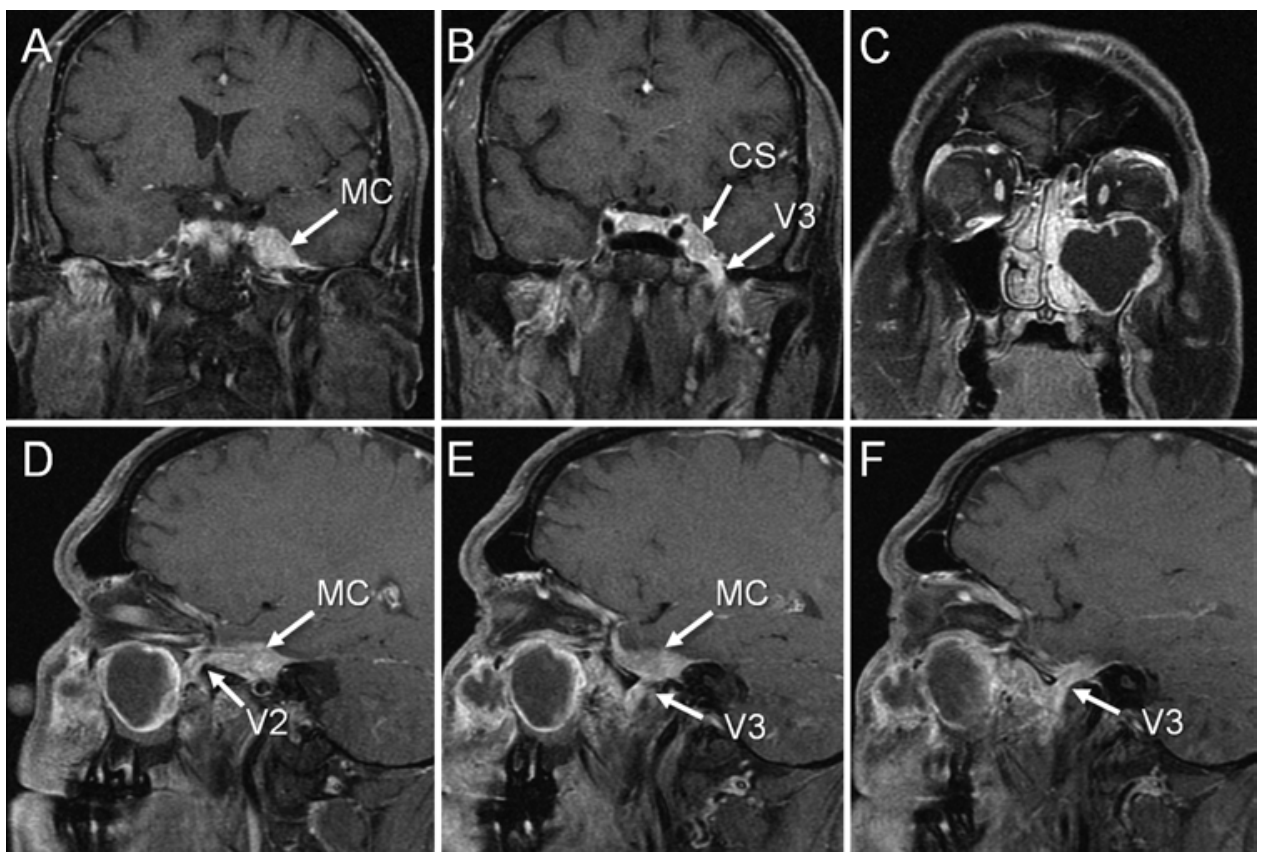

FIG. 2. Preoperative coronal (A-C) and sagittal (D-F) T1-weighted Gd-enhanced MR images demonstrating an enhancing left cavernous sinus (CS) mass that extends into the Meckel cave (MC), V2 through the foramen rotundum, and V3 through the foramen ovale. The tumor extends along the infraorbital nerve toward the maxilla, with a cystic component in the left maxillary sinus $(\mathrm{C}-\mathrm{F})$.

extension into the foramen ovale and foramen rotundum. There was a giant cystic component extending into the maxillary sinus along the course of the infraorbital nerve toward the maxillary bone of the face (Fig. 2). The initial differential diagnosis included trigeminal schwannoma. The patient did not have any stigmata of NF1 or NF2 .

To achieve maximal resection of the tumor, a staged transcranial and transfacial approach was performed. In the first stage, the patient underwent a left frontotemporal transcavernous extradural approach to resect the tumor from the cavernous sinus and Meckel cave. The temporal dura and lateral wall of the cavernous sinus was elevated extradurally to expose the tumor infiltrating the trigeminal ganglion, V2, and V3. The tumor in this region was removed, with care taken to preserve CN III, CN IV, and V1.

One month later, the patient underwent second-stage transfacial resection of the extracranial portion of tumor. An endoscope-assisted left medial maxillectomy was performed via a combined endonasal and sublabial Caldwell-Luc approach to the left infratemporal fossa using a Weber-Ferguson incision. Tumor was removed from the nasal cavity, maxillary sinus, and infratemporal fossa. The tumor extended along the course of the infraorbital nerve at the roof of the maxillary sinus and eroded the maxilla within the face. There was significant difficulty obtaining negative bony margins without sacrificing crucial surrounding structures and the preliminary pathological report suggested that the tumor was an MPNST. Further resection then was stopped in favor of postoperative adjuvant radiotherapy.

Postoperative MRI revealed minimal residual tumor in the cavernous sinus (Fig. 3). The remainder of the hospital course was unremarkable, and the patient was discharged in stable neurological condition without any CSF leakage. Extraocular muscle function improved to normal after decompression of the cavernous sinus. Postoperative trigeminal neuropathic pain was controlled with gabapentin. Pathological examination showed a diffusely infiltrative tumor with elongated nuclei exhibiting extensive involvement of peripheral nerve tissue and myelinated fibers (Fig. 4A). Immunohistochemical staining was strongly positive for S-100 (Fig. 4B), consistent with a diagnosis of MPNST. The patient underwent 5940 cGy of radiation therapy and was alive with stable disease in the cavernous sinus 6 months after surgery.

\section{Discussion}

\section{Malignant Peripheral Nerve Sheath Tumors}

Malignant peripheral nerve sheath tumors are rare neoplasms of peripheral Schwann cells, with an incidence of $0.001 \%$ in the general population..$^{9} 12$ The majority of these cases involve the trunk and extremities. ${ }^{31}$ There is generally no sex or race predilection, but MPNSTs are more commonly found in older patients. ${ }^{12}$ Although it is widely accepted that uncontrolled growth of Schwann cells is the cause of these tumors, it has been suggested that they occur de novo and do not arise as a result of malignant transformation from a benign schwannoma. ${ }^{1,3,23,31,33}$ However, some reports have suggested that radiation may play a role in the development of MPNSTs. ${ }^{22}$ The presence of NF greatly increases the odds of developing MPNSTs, and patients with NF1 are over 4000 times more likely to develop an MPNST in their lifetime than individuals without this condition. Patients with NF 

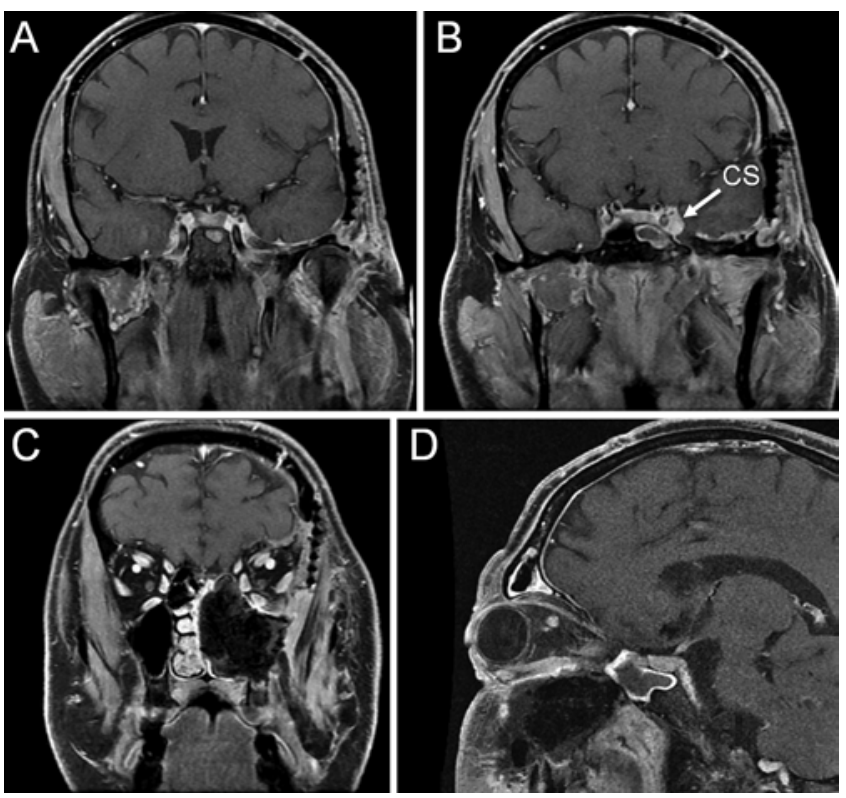

FIG. 3. Postoperative coronal (A-C) and sagittal (D) T1-weighted Gd-enhanced MR images demonstrating interval removal of the mass within the Meckel cave, V2, V3, and maxillary sinus. There is residual tumor in the left cavernous sinus.

also typically have a much younger age of onset..$^{9,12,31} \mathrm{Al}$ though the presence of NF is known to greatly increase the odds of developing MPNSTs in general, it is interesting to note that it has not been shown to play a significant role in MPNSTs of the cranial nerves., ${ }^{1,33}$ Of the reports we identified for patients with a trigeminal MPNST, only one patient was described as having NF1 and one as having NF2. ${ }^{15}$ However, our study is limited in that not all of the cases we reviewed specified whether the patient had a history of NF, and some studies specifically excluded patients with a history of NF.

Malignant peripheral nerve sheath tumors comprise $2 \%-6 \%$ of head and neck sarcomas. ${ }^{22}$ Gender has been implicated as playing a small role in the development of intracranial MPNSTs, unlike other MPNSTs, with a higher incidence reported in men. ${ }^{4}$ Our report of trigeminal MPNSTs corroborates this suggestion, as we found $77.1 \%$ of the patients in our analysis to be male. Previously, MPNSTs of the cranial nerves were suggested to arise more often in older patients. Our study showed an average patient age of 44.6 years, with a wide range (8-71 years).

As seen in our study, it is not uncommon for MPNSTs of the trigeminal nerve to involve more than one branch. It has been suggested that multiple branch involvement is characteristic of trigeminal MPNSTs originating from the gasserian ganglion, with this origin enabling them to spread through multiple divisions of the nerve. ${ }^{23,34}$ While we found that the MPNSTs did not necessarily originate in the gasserian ganglion in all patients who had involvement of multiple branches, gasserian ganglion involvement may have been underreported. Trigeminal MPNSTs have also been suggested to spread from postganglionic divisions back to the gasserian ganglion. ${ }^{23}$ The ophthalmic branch of the trigeminal nerve is implicated as a common site of origin for retrograde growth; however, it

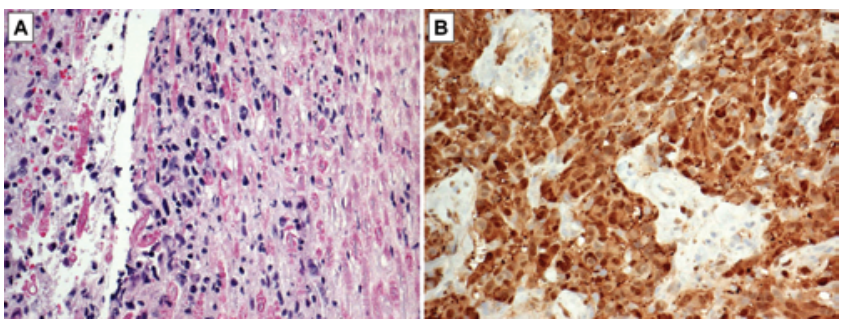

Fig. 4. Histopathology of MPNST. A: An H \& E-stained section of the tumor specimen obtained from the Meckel cave. The tumor cells exhibit extensive infiltration of the peripheral nerve and myelin sheaths (stained red). B: Immunohistochemical stain for S-100 shows tumor cells with strong positive staining, highly indicative of a malignant peripheral nerve sheath tumor. Original magnification $\times 40(A$ and $B)$.

was the least involved branch identified in this review and only a fraction of patients with a V1 origin also had gasserian ganglion involvement.

\section{Clinical Presentation}

Trigeminal MPNSTs can be hard to diagnose on the basis of clinical presentation, because their clinical presentation mimics that of a benign schwannoma of the trigeminal nerve. ${ }^{14,33,34}$ In our review we found a wide variety of presenting symptoms. The majority of patients presented with altered facial sensation and/or facial pain distributed along the affected divisions. ${ }^{1,4,6,7,11,14,16,17,20,21,24,33,37}$ Furthermore, weakness of the mastication muscles was also seen in a number of patients. ${ }^{17,20,33}$ The involvement of other cranial nerves was relatively common among the population we studied, and we found that it was typically due to spread of malignant tumor through the cavernous sinus and other intracranial or extracranial compartments. Diplopia and facial weakness were the most common of complaints related to involvement of other cranial nerves. Diplopia is expected to be seen in tumors involving the cavernous sinus, as tumor involvement here can affect the nerves coursing through the sinus., ${ }^{1,5,11,16,20,24}$ Hearing loss was also seen, often as the result of local compression or nerve destruction in posterior fossa tumors. ${ }^{7,17,19}$ Distant metastasis of trigeminal MPNSTs was also reported, with one patient having involvement of the clavicle. ${ }^{11}$

Currently, numerous methods exist to guide the diagnosis of MPNSTs. Magnetic resonance imaging can be useful, but it is difficult to discern between benign and malignant tumors based on imaging. ${ }^{4,12}$ On the other hand, some have suggested that bony erosion on CT or plain radiographs favors malignancy over a benign tumor. ${ }^{4}$ Typically, the diagnosis of these tumors requires pathological analysis of biopsy specimens. Details regarding the pathological findings of these tumors have been well established, and discussion of these details is beyond the scope of this paper. However, markers such as S-100, Leu7 , and myelin basic protein are frequently used to aid in diagnosing these lesions. ${ }^{33}$ In particular, an elevated ratio of S-100 $\alpha$ to S-100 $\beta$ favors malignancy over a benign etiology. ${ }^{1}$ Additionally, the absence of S-100 immunoreactivity altogether may indicate dedifferentiation of tumor cells. ${ }^{1,16}$ Nevertheless, it is important to remain cautious when using immunohistochemical staining as the sole di- 


\section{Malignant peripheral nerve sheath tumors of the trigeminal nerve}

agnostic criterion of MPNSTs, as they are typically associated with high false-positive and false-negative rates. ${ }^{12}$

\section{Prognosis and Treatment}

In general, MPNSTs carry a poor prognosis. Looking at studies of all MPNSTs revealed that local recurrence rates are generally very high, ranging from $30 \%$ to $60 \%,{ }^{12}$ and the tumors recur even in cases of complete resection. ${ }^{5}$ MPNSTs have also been shown to metastasize to the lungs, soft tissues, bone, liver, and/or brain nearly $65 \%$ of the time. , $^{4,12,31}$ The 5-year survival rate for patients with these tumors ranges from $10 \%$ to $50 \%$, and patients with a history of NF have an exceptionally poor prognosis. . $^{1,5,9,12,22,31}$ When only MPNSTs of the head and neck are considered, the 5-year survival rate decreases to about $20 \% .^{25}$ The limited nature of this analysis and poor consistency of follow-up makes it difficult to offer specific prognostic factors for trigeminal MPNSTs; however, it is clear that they tend to exhibit similar features of very rapid growth and extensive infiltration into surrounding tissues, which results in a generally grim prognosis. ${ }^{4}$

Due to the aggressive natural history of these tumors and the more favorable outcomes seen with complete resection, ${ }^{4,5,37}$ aggressive radical resection of trigeminal MPNSTs should be attempted, if safely possible. Recent advances in microsurgical and endoscopic techniques have allowed the potential for larger exposures, enabling more extensive resections of previously unreachable or unresectable tumors. However, these tumors are highly infiltrative and usually involve critical surrounding structures, such as the cavernous sinus, internal carotid artery and the orbit. Therefore, the ability to obtain a total resection may be difficult. In our review, a wide variety of approaches were used, including frontotemporal, subtemporal, pterional, transnasal, transfacial, and posterior fossa approaches. In our patient, we used a staged approach, with an initial frontotemporal extradural transcavernous approach followed by an endoscope-assisted transfacial approach. Our resection was limited by residual disease within the cavernous sinus making total resection unfeasible without causing unacceptable deficits and patient morbidity.

The use of radiation therapy for MPNSTs has been debated,, 912 but more recent reports suggest that these tumors are radiosensitive and that radiation offers increased rates of tumor control and long-term survival. ${ }^{25}$ Currently, the established method of treatment for MPNSTs involves aggressive resection followed by radiation therapy.,37 Due to the highly malignant nature of these tumors, radiation therapy is typically administered even in cases of complete resection. ${ }^{4}$ This is supported by our study: all 6 of the patients who underwent complete resection followed by radiation therapy were reported to show no evidence of recurrence throughout the follow-up period. Due to the relatively poor outcomes with patients treated by radiation therapy alone, we do not recommend this modality in patients who are good surgical candidates. However, it may be appropriate in patients where resection is not feasible due to their clinical status. The role of chemotherapy is still not well defined and the reports that exist are not yet conclusive. However, it may be appropriate as an adjuvant therapy in certain scenarios, such as patients with metastatic disease or systemic pathology, where surgical intervention or radiation alone might offer limited therapeutic benefit. In general, maximal resection, if feasible, followed by postoperative radiation therapy should be considered in all patients, as the availability of advanced surgical techniques has allowed access to a number of lesions that were previously unreachable and complete resection is associated with the best outcomes.

\section{Study Limitations}

There are numerous limitations to this study. Primarily, the general lack of existing literature regarding MPNSTs of the trigeminal nerve limits the extent of statistical analysis that can be made due to the small sample size. Furthermore, in this study there was no method of standardization for reporting of cases. Many cases are missing information regarding the specifics of clinical appearance, extent of tumor involvement, the radiological tests that were performed, the modes of treatment that were undertaken, and the presence or absence of NF. Additionally, reporting of follow-up and long-term patient outcomes was largely inconsistent. Many of the studies were marred by short follow-up times, where positive patient outcomes may be likely to be reported. The treatment strategies were also heterogeneous. Many of the cases presented in this study also occurred before the widespread use of modern diagnostic and therapeutic interventions, such as MRI, stereotactic radiosurgery, operative microscopy, and endoscopic approaches. As such, the outcomes of previous studies may not be comparable to those of current cases, given the technologies that are available today. However, we believe that this study remains the most thorough analysis of this disease to date, offering an unprecedented look at the clinical appearance, treatment options, and outcomes of this rare disease.

\section{Conclusions}

Malignant peripheral nerve sheath tumors are relatively rare tumors, with only a very small subset of these lesions arising from the trigeminal nerve. Patients with MPNSTs present with a variety of clinical manifestations; however, the most common are facial pain and/or numbness of the affected nerve branches. Due to their highly infiltrative nature, relatively high rates of recurrence, and generally poor prognosis, aggressive resection is the treatment of choice for these tumors, with complete resection being the ultimate goal, followed by adjuvant radiation therapy.

\section{Disclosure}

The authors report no conflict of interest concerning the materials or methods used in this study or the findings specified in this paper.

Author contributions to the study and manuscript preparation include the following. Conception and design: Liu, Eloy. Acquisition of data: all authors. Analysis and interpretation of data: Liu, Schmidt, Yick, Boghani. Drafting the article: Schmidt, Yick, Boghani. Critically revising the article: Liu, Schmidt, Eloy. Reviewed submitted version of manuscript: all authors. Approved the final version of the manuscript on behalf of all authors: Liu. Statistical analysis: Schmidt. Study supervision: Liu. 


\section{Acknowledgment}

The authors would like to thank Dr. Leroy Sharer for providing the histopathological images in this manuscript.

\section{References}

1. Akimoto $\mathrm{J}$, Ito $\mathrm{H}$, Kudo M: Primary intracranial malignant schwannoma of trigeminal nerve. A case report with review of the literature. Acta Neurochir (Wien) 142:591-595, 2000

2. Bailet JW, Abemayor E, Andrews JC, Rowland JP, Fu YS, Dawson DE: Malignant nerve sheath tumors of the head and neck: a combined experience from two university hospitals. Laryngoscope 101:1044-1049, 1991

3. Beauchesne P, Mosnier JF, Schmitt T, Brunon J: Malignant nerve sheath tumor of the right cerebral peduncle: case report. Neurosurgery 54:500-504, 2004

4. Bowers CA, Taussky P, Duhon BS, Chin SS, Couldwell WT: Malignant peripheral nerve sheath tumour of the trigeminal nerve: case report and literature review. Br J Neurosurg 25: 750-753, 2011

5. Chibbaro S, Herman P, Povlika M, George B: Malignant trigeminal schwannoma extending into the anterior skull base. Acta Neurochir (Wien) 150:599-604, 2008

6. Cuneo HM, Rand CW: Tumors of the gasserian ganglion; tumor of the left gasserian ganglion associated with enlargement of the mandibular nerve; a review of the literature and case report. J Neurosurg 9:423-431, 1952

7. DeRubeis DA, Woulfe J, Rosso D, Lownie S, Parnes L, Lee D, et al: Case of the month: June 1997-a 42 year old man with left facial weakness. Brain Pathol 8:233-234, 1998

8. Dinakar I, Rao SB, Rao KS: Malignant schwannoma of the trigeminal nerve. Neurol India 19:43-44, 1971

9. Ducatman BS, Scheithauer BW, Piepgras DG, Reiman HM, Ilstrup DM: Malignant peripheral nerve sheath tumors. A clinicopathologic study of 120 cases. Cancer 57:2006-2021, 1986

10. Fisher BJ, Dennis KE: Malignant epithelioid cranial nerve sheath tumor: case report of a radiation response. J Neurooncol 78:173-177, 2006

11. Ganapathy K, Ghosh M: Clavicular metastasis from malignant trigeminal schwannoma. J Clin Neurosci 10:485-492, 2003

12. Gupta G, Mammis A, Maniker A: Malignant peripheral nerve sheath tumors. Neurosurg Clin N Am 19:533-543, 2008

13. Hajdu SI: Peripheral nerve sheath tumors. Histogenesis, classification, and prognosis. Cancer 72:3549-3552, 1993

14. Hedeman LS, Lewinsky BS, Lochridge GK, Trevor R: Primary malignant schwannoma of the Gasserian ganglion. Report of two cases. J Neurosurg 48:279-283, 1978

15. Higami Y, Shimokawa I, Kishikawa M, Okimoto T, Ohtani $\mathrm{H}$, Tomita M, et al: Malignant peripheral nerve sheath tumors developing multifocally in the central nervous system in a patient with neurofibromatosis type 2. Clin Neuropathol 17: 115-120, 1998

16. Horie Y, Akagi S, Taguchi K, Yoshino T, Hayashi K, Takahashi $\mathrm{K}$, et al: Malignant schwannoma arising in the intracranial trigeminal nerve. A report of an autopsy case and a review of the literature. Acta Pathol Jpn 40:219-225, 1990

17. Karmody CS: Malignant schwannoma of the trigeminal nerve. Otolaryngol Head Neck Surg (1979) 87:594-598, 1979

18. Khademi B, Owji SM, Khosh KJ, Mohammadianpanah M, Gandomi B: Description of a neural sheath tumor of the trigeminal nerve: immunohistochemical and electron microscopy study. Sao Paulo Med J 124:333-335, 2006

19. Lee JH, Lee HK, Choi CG, Suh DC, Lee KS, Khang SK: Malignant peripheral nerve sheath tumor in the parapharyngeal space: tumor spread through the eustachian tube. AJNR Am J Neuroradiol 22:748-750, 2001

20. Levy WJ, Ansbacher L, Byer J, Nutkiewicz A, Fratkin J: Primary malignant nerve sheath tumor of the gasserian ganglion: a report of two cases. Neurosurgery 13:572-576, 1983
21. Liwnicz BH: Bilateral trigeminal neurofibrosarcoma. Case report. J Neurosurg 50:253-256, 1979

22. Loree TR, North JH Jr, Werness BA, Nangia R, Mullins AP, Hicks WL Jr: Malignant peripheral nerve sheath tumors of the head and neck: analysis of prognostic factors. Otolaryngol Head Neck Surg 122:667-672, 2000

23. MacNally SP, Rutherford SA, Ramsden RT, Evans DG, King AT: Trigeminal schwannomas. Br J Neurosurg 22:729-738, 2008

24. Maroun FB, Sadler M, Murray GP, Mangan M, Mathieson G, Jacob JC, et al: Primary malignant tumours of the trigeminal nerve. Can J Neurol Sci 13:146-148, 1986

25. Minovi A, Basten O, Hunter B, Draf W, Bockmühl U: Malignant peripheral nerve sheath tumors of the head and neck: management of 10 cases and literature review. Head Neck 29: 439-445, 2007

26. Nakamura T, Ono T, Yoshimura K, Arao T, Kondo S, Ichihashi M, et al: Malignant schwannoma associated with xeroderma pigmentosum in a patient belonging to complementation group D. J Am Acad Dermatol 25:349-353, 1991

27. Ohnishi M, Tanaka Y, Tutui T, Bann S: Extensive malignant schwannoma of the mandibular nerve. Case report. Int J Oral Maxillofac Surg 21:280-281, 1992

28. Robertson I, Cook MG, Wilson DF, Henderson DW: Malignant schwannoma of cranial nerves. Pathology 15:421-429, 1983

29. Rodríguez JA, Hedges TR III, Heilman CB, Strominger MB, Laver NM: Painful sixth cranial nerve palsy caused by a malignant trigeminal nerve sheath tumor. J Neuroophthalmol 27:29-31, 2007

30. Sempere-Ortega C, Martinez-San-Millan J: Perineural invasion through the maxillary division of the right trigeminal nerve in a rare case of nasolabial malignant peripheral nerve sheath tumor. AJNR Am J Neuroradiol 29:396-397, 2008

31. Stark AM, Buhl R, Hugo HH, Mehdorn HM: Malignant peripheral nerve sheath tumours-report of 8 cases and review of the literature. Acta Neurochir (Wien) 143:357-364, 2001

32. Stark AM, Buhl R, Hugo HH, Straube T, Mehdorn HM: Chronic recurrent subarachnoid hemorrhage from a trigeminal nerve malignant peripheral nerve sheath tumor: case report. Neurosurgery 59:E425, 2006

33. Stone JA, Cooper H, Castillo M, Mukherji SK: Malignant schwannoma of the trigeminal nerve. AJNR Am J Neuroradiol 22:505-507, 2001

34. Tegos S, Georgouli G, Gogos C, Polythothorakis J, Sanidas V, Mavrogiorgos C: Primary malignant schwannoma involving simultaneously the right Gasserian ganglion and the distal part of the right mandibular nerve. Case report. J Neurosurg Sci 41:293-297, 1997

35. Ueda R, Saito R, Horiguchi T, Nakamura Y, Ichikizaki K: Malignant peripheral nerve sheath tumor in the anterior skull base associated with neurofibromatosis type 1-case report. Neurol Med Chir (Tokyo) 44:38-42, 2004

36. Yamashiro S, Nagahiro S, Mimata C, Kuratsu J, Ushio Y: Malignant trigeminal schwannoma associated with xeroderma pigmentosum-case report. Neurol Med Chir (Tokyo) 34: 817-820, 1994

37. Ziadi A, Saliba I: Malignant peripheral nerve sheath tumor of intracranial nerve: a case series review. Auris Nasus Larynx 37:539-545, 2010

Manuscript submitted November 13, 2012.

Accepted November 29, 2012.

Please include this information when citing this paper: DOI: 10.3171/2012.11.FOCUS12292.

Address correspondence to: James K. Liu, M.D., Center for Skull Base and Pituitary Surgery, Department of Neurological Surgery, UMDNJ-New Jersey Medical School, 90 Bergen Street, Suite 8100, Newark, New Jersey 07103. email: james.liu@umdnj.edu. 